\title{
Review of: "Impact of the COVID-19 pandemic on the mental health of professionals in 77 hospitals in France"
}

Annalisa Boscolo

Potential competing interests: The author(s) declared that no potential competing interests exist.

Dear Editor,

it's a pleasure to revise this article focused on the impact of the pandemic on the mental health of professionals working in health care institutions and on environmental factors influencing the risk of mental health disorders. The authors concluded that about $57 \%$ of the professionals suffered from psychological distress, and $21 \%$ showed symptoms of potential post-traumatic stress with some differences between wards. Additionally, support services and management strategies within hospitals are recommended with the aim to underline the importance of positive thinking and social support.

According to this, the manuscript is well written, statistical analysis performed properly, depsite a missing sample size (could you provide it?).

Additionally, the questionary was not administrated to students or residents, could you explain why? Moreover, the addition of this reference is recommended PMID: 33525201.

the manoscript needs some minor revisions. 